\title{
MATHEMATICAL EXPANSION OF SPECIAL THEORY OF RELATIVITY ONTO ACCELERATIONS
}

\author{
Jakub Czajko \\ Science/Mathematics Education Department \\ Southern University and A\&M College, Baton Rouge, LA 70813, USA \\ Email : sunswing77@netscape.net
}

\begin{abstract}
The special theory of relativity (STR) is operationally expanded onto orthogonal accelerations: normal $a_{N}$ and binormal $a_{B}$ that complement the instantaneous tangential speed $v_{T}$ and thus can be structurally extended into operationally complete 4D spacetime without defying the STR. Thus the former classic Lorentz factor, which defines proper time differential $d \tau=\sqrt{1-\frac{v^{2}}{c^{2}}} d t \quad$ can $\quad$ be $\quad$ expanded $\quad$ onto $\quad d \tau=\frac{1}{c} \sqrt{c^{2}-v_{T}^{2}-v_{N}^{2}-v_{B}^{2}} d t=$ $\frac{1}{c} \sqrt{c^{2}-v_{T}^{2}-\left(t_{N} a_{N}\right)^{2}-\left(t_{B} a_{B}\right)^{2}} d t$ within a trihedron moving in the Frenet frame $(\mathbf{T}, \mathbf{N}, \mathbf{B})$. Since the tangential speed $v_{T}$ which was formerly assumed as being always constant, expands onto effective normal and binormal speeds ensuing from the normal and binormal accelerations, the expanded formula conforms to the former Lorentz factor. The obvious though previously overlooked fact that in order to change an initial speed one must apply accelerations (or decelerations, which are reverse accelerations), made the Einstein's STR incomplete for it did not apply to nongravitational selfpropelled motion. Like a toy car lacking accelerator pedal, the STR could drive nowhere. Yet some scientists were teaching for over 115 years that the incomplete STR is just fine by pretending that gravity should take care of the absent accelerator. But gravity could not drive cars along even surface of earth. Gravity could only pull the car down along with the physics that peddled the nonsense while suppressing attempts at its rectification. The expanded formula neither defies the STR nor the general theory of relativity (GTR) which is just radial theory of gravitation. In fact, the expanded formula complements the STR and thus it supplements the GTR too. The famous Hafele-Keating experiments virtually confirmed the validity of the expanded formula proposed here.
\end{abstract}

Keywords: Expanded special relativity, special-relativistic accelerations, univocal special relativity

\section{INTRODUCTION}

Some remarks are so impressive that one may feel compelled to reevaluate afresh everything we learned heretofore. One of such profound remarks to me is that if physical laws evolve in time, then time must play a preferred role in the world; it cannot be just another dimension and it cannot just disappear in a timeless block universe or "wavefunction of the universe" [1]. In conjunction with the assertion that superspace is not a manifold but a stratified union of manifolds and spacetime is a sheaf of geodesics in the superspace [2] one may wonder what the abstract mathematical time really is. Other profoundly innovative to me assertions were that mass and electrostatic potential, which were known as scalars, are found to be vectors in time and that scalar potential present in 4D Lorentz equation should be [represented by] a contravariant vector in time [3] and that 4D world is spherical with respect to space but 
hyperboloidal with respect to the [elapsing] time axis [4]. Such statements (and many others, of course) prompted me to reexamine several operational formulas of mathematics and reassess the scope of validity of some physical laws and principles which utilized the traditional mathematics that now seems controversial to me.

Traditional mathematics operated under several operationally deficient and structurally questionable principles, which were not only unchallenged but oftentimes fiercely defended against logic and in defiance of operational and/or structural laws that were proven and widely accepted. The resulting mathematical lies then spilled into physics where they led to creation of various tacitly veiled nonsenses, which were then propagated with impunity and against the common scientific sense and sometimes even with open disrespect for reason.

Insofar as I am concerned, the most blatant violations of mathematics are the - obviously infantile - prohibition of division by zero enacted by unjustifiable decree, and the unwarranted single space reality (SSR) paradigm which should be promptly replaced by the multispatial reality (MSR) paradigm with the help of which I was able to reconcile few formerly unreconciled and even deemed as unreconcilable results of some unbiased - yet previously quite unanticipated - physical experiments.

We are taught that science was built by accumulation of observed theoretical and/or experimental facts, invented or derived from principles (obtained by generalizations of the facts), which then are abstracted and proven as laws or just operational rules. But experiments usually confirm only certain most discernible facts while urging us to discard some principles used to develop the theories that predicted the very experiments. Apparently, the Nature is much more complex than our mathematically challenged imagination could fathom.

Paul Duhem already asked the question if the principle of universal gravitation is merely a generalization of Kepler's laws and their extension onto the motion of satellites. And he concluded that the Newton's principle of universal gravitation cannot be derived from Kepler's laws, but it actually does contradict the latter laws [5].

That is why I have developed new synthetic approach to mathematics and mathematized sciences, which emphasizes the need to synthesize the previously unfathomable features of our mathematical and physical reality from confrontation of apparent or actual contradictions. We cannot just stick forever to previously helpful principles which - nevertheless - could become obsolete by more sophisticated experiments predicted by theories built upon these principles. Besides, principles are just steppingstones on our convoluted ways to grasp the often bewildering us features of the Nature. Even rigorously proven scientific laws may need some conceptual adjustments, which usually are triggered by shifting paradigms.

In this paper I intend to demonstrate that the classic, formerly incomplete special theory of relativity (STR) can be made operationally complete. Impacts of the expanded onto accelerations STR on the general theory of relativity (GTR) shall be discussed elsewhere.

\section{SUMMARY OF THE GOAL AND OBJECTIVES OF THIS PAPER}

The main goal of the present paper is to present mathematically inspired enhancement of the classical special theory of relativity. The motivation for writing this paper was the fairly recent crash of the SJ Air flight182, which might have been prevented, I think. My primary objective is to recast my theoretical paper, which was accepted and scheduled for publication in Germany, but was then retroactively rejected by next editor-in-chief, presumably because I have derived an expansion of the original Lorentz $\gamma(v)$ factor $d \tau=\frac{d t}{\gamma(v)}=\sqrt{1-\frac{v^{2}}{c^{2}}} d t$ onto radial acceleration, which was - and still is in some scientific circles - anathema. Since the 
original Lorentz $\gamma$ factor looks like a toy car without an accelerator, which can be used as a calculational device but could not really drive on an even surface, it was plausible to expect that a more realistic functional of the form: $d \tau=\frac{d t}{\gamma(v, a)}=\sqrt{1-\frac{v^{2}}{c^{2}}-\frac{(a \Delta t)^{2}}{c^{2}}} d t$ should be proposed, which I already did over 30 years ago [42]. My other objective is to keep the - still incomplete on purpose - expanded formula in compliance with results of the Hafele-Keating experiments, from which the acceleration-dependent expansion was inferred back then. Thus, a complete formula shall be offered in my next paper on the topic, where it is extended into all three components of the total/complete acceleration.

\section{LAPLACE EQUATION IS WELL-POSED IN CARTESIAN COORDINATES BUT APPEARS ILL-POSED IN CURVED COORDINATES}

Differential equations are said to be well-posed if their solutions exist, are unique and are considered stable, as stable solutions depend continuously on boundary conditions and initial conditions [6]. Otherwise, the equations are deemed as ill-posed. For some general issues pertaining to stability of solutions of functional equations see [7].

Glasko pointed out that being ill-posed in the elementary formulation is the characteristic property of inverse problems [8]; compare also [9], [10]. For some nonlinear illposed problems see [11]. For examples of inverse problems in the SSR setting see [12].

The Glasko's remark linking ill-posed problems to inverse representations of differential equations could be the most profound realization thus far that perhaps something is really wrong with the abstract notion of 'inversion' as it is interpreted in traditional mathematics. If the links of inverses to ill-posed problems were seriously investigated, and the connection between invertibility and ill-posedness reviewed without bias, we might have realized long ago that we often mix two quite distinct concepts when we use additive reverse instead of multiplicative inverse [13]. Since mutually dual reciprocal spaces are multiplicatively inverse by definition, the problems investigated within the abstract multispatial realm of such reciprocal spatial structures could be considered as ill-posed in the traditional singlespatial reality framework, of course. But they may also appear as being wellposed once reconsidered under the auspices of the MSR paradigm, provided that the differential equations are properly expressed in dual reciprocal spaces. This is because twin reciprocal spaces are truly multiplicatively inverse, and it is known that certain finegrained differential operators [14] act simultaneously on objects represented in both: the primary space and its dual reciprocal space [15]. For abstract pairing of spaces see [16]. For the new product differentiation rule that facilitates the pairing see [17]. For finegrained differential operators see [14]. By the way, I shall be crossing out the definite article wherever it can lead to confusion, for even repeated usage of the phrase does not contribute anything of merit to understanding of the new concept whose full reverberation is still too far away to make some of its previously unfathomable predictions foreseeable indeed. Expressions used in mathematics and mathematized sciences should not be governed by linguistic colloquiality. The only things that matter here to me are unambiguous precision of mathematical (i.e. both operational and structural) formulation of scientific expressions and their truth.

Having said that, let us consider the Laplace's differential equation in 2D plane:

$$
\nabla^{2} u(x, y):=u_{x x}+u_{y y} \Leftrightarrow u_{r r}+\frac{1}{r} u_{r}+\frac{1}{r^{2}} u_{\theta \theta}=: \nabla^{2} u(r, \theta)
$$


pictured in the 2D rectangular coordinates $(\mathrm{x}, \mathrm{y})$ on the left-hand side LHS of the equivalence (1) and in the 2D polar coordinates $(r, \theta)$ on the RHS - compare [18] p.403; one may see also [19] for Laplace equation evaluated in both 2D and 3D representations.

Since there is no question that inverse problems are deemed as being ill-posed in the formerly unspoken traditional SSR setting - as Glasko and some other scientists duly acknowledged - then why is the very same 2D Laplace equation seemingly well-posed on the LHS of the equivalence (1) but appears as ill-posed on the RHS, apparently depending on the coordinate system used for its depiction? Moreover, if the ill-posedness does depend on coordinate representation then which particular attribute of the polar coordinate system causes the curious failure of the acknowledged well-posedness persisting in rectangular coordinate system that actually turns it into definitely recognizable ill-posedness on the allegedly unproblematic transition to the - formally equivalent - polar coordinate system?

If not the notion of ill-posedness then perhaps the concept that underlies the notion causes the cancellation of well-posedness during transition from the flat rectangular coordinates to the curved polar coordinates. Notice that dimensionality remains the same. Or is it the potential function $\mathrm{u}()$,_upon which the Laplacian acts, that causes the dilemma?

Obviously the ill-posedness of the polar representation of the - otherwise well-posed, if represented in rectangular coordinates - Laplace equation is linked to the multiplicatively inverse radial terms $\frac{1}{r}$ and $\frac{1}{r^{2}}$ appearing in the polar representation of the equation, but the question to be answered is this: are the inverse terms the actual cause of the ill-posedness or perhaps they are just symptoms of a much deeper cause of that conceptual illness besetting the traditional mathematics that was developed under the SSR paradigm? Since we cannot cure the mathematical malady by just suppressing its symptoms, we are thus obliged to pinpoint and explore its underlying causes before we propose a realistic cure.

I do not have any problem with acceptance of the aforesaid definition of well-posedness or any of its equivalents. The ill-posedness is also too trivial a notion to be wrong in practice or in theoretical considerations. Thus, I would be unequivocally against any presumptive redefinition of the simple notion of well-posedness, because even well-meant redefinition might merely conceal the above controversy exemplified in (1). Neither would I advocate watering down the - corresponding to it - notion of ill-psedness nor reformulate it so that the traditional mathematics could be viewed as perfect insofar as its internal consistency is concerned. We must face the truth that the inconsistency permitting the Laplace equation to be both well-posed as well as ill-posed depending on the - allegedly quite equivalent representations urges reevaluation of most fundamental paradigms underlying mathematics.

Judging by its relevance to the whole issue, perhaps it is the formerly unspoken (and thus unchallenged, even though it is not really selfevident nor easily noticeable) SSR paradigm that is actually ill-conceived, because it evidently permits us to maintain the alleged formal equivalence of the rectangular and polar representations of the same differential equation. And yet in the SSR setting we just cannot unambiguously explain the lingering controversy between the two naturally contradictory notions of well-posedness and ill-posedness.

The whole controversy is actually caused by the - heretofore ignored yet clearly visible to me - fact that the modulus/radius $r$ (in complex-analytic parlance) and the argument/angle $\theta$ of the polar and spherical polar coordinates are not quite compatible when it comes to their mode of varying. The radius is multiplicatively inversive variable whereas the angle is merely an additively reversive magnitude - compare my critique of analytic functions, whose theory is not always incorrect, but it is certainly conceptually inconsistent [13]. Their incompatible varying was one of the main operational reasons for me to come up with the idea of pairing of dual reciprocal algebraic spaces as well as pairing of mutually reciprocal (geometric and/or 
quasigeometric) spatial structures. Nevertheless, the still dangling question to be answered is: Am I justified to blame the undeniable controversy shown in (1) on the incompatible modes of varying of independent variables? Or is it perhaps just a misunderstood insignificant nuance of an operational (or geometric) dimensionality or both that causes the whole controversy at a deeper conceptual level?

Some authors used to argue that the scalar potential function $u(r, \theta)$ featured in Laplace equation presented in spherical coordinates can only be a function of the radius $r$ since there is no way to distinguish a point $(r, \theta, \phi)$ from another point with the same $r$ but different [angles] $\theta$ and $\phi$ see [20], for instance. This kind of sophistry makes alert students cringe for the dilemma is apparently nonexistent in rectangular coordinate system, even though it was unexplored before I have demonstrated the (suggested by certain experiments) possibility of presence of some nonradial components of the potential in [21]. In the meantime I have showed that other than radial effects of purely radial force fields do exist indeed [22] even though they depend inversely on density of matter as well [23], [24], [25], [26]; see also [27] for theoretical necessity of presence of nonradial effects. Recall that density of matter is not the same as density of mass, which is motion-related inertial attribute of matter. See [26] for discussion of the (experimentsally confirmed in [21]) difference which is explained in [28].

The general conclusion to be drawn from the above reasonings is that curved coordinates are not really equivalent to the rectangular coordinates, even though variables and functions can be converted between these coordinate systems. Moreover, coordinatefree representations not only conceal several features of objects treated by tensor calculus, which is fundamentally flawed [29] even though its flaws were cleverly concealed while its afficionados preventd them from being uncovered by suppression of scientific papers whose aim was to rectify the flaws. Those insidiously perpetuated flaws covered up many nonsenses that may be difficult to perceive in mathematics but are fairly easy to grasp in physics backed by experiments. Recall that metric tensor is not an intrinsic part of the spacetime manifold, but just an object which lives in the spacetime [30]. It is thus a tensorial representation of the object. Therefore, I am going to expose in this present paper some easy to recognize nonsenses by appeal not only to mathematics but also to results of few unbiased physical experiments..

\section{HAFELE-KEATING EXPERIMENTS DEMAND OPERATIONAL EXPANSION OF THE EINSTEIN'S SPECIAL THEORY OF RELATIVITY}

The Hafele-Keating experiments with four atomic clocks flown around-the-world aboard commercial airplane virtually confirmed the need to employ accelerations in order to account for the East-West asymmetry found by both Hafele as well as by Alley who conducted similar experiments. For after all effects predicted by the GTR and STR [31] have been taken into account, there still remained some then impossible to explain residual discrepancy between the Eastbound and Westbound flights [32]. Similar East-West discrepancy has been found by Alley who also conducted experiments with atomic clock flown aboard an airplane [33]. What is even more important to me, is that when Alley sent the airplane carrying the atomic clock in the practically South-North direction (between Washington, DC, and Thule, Greenland) and back, no such discrepancy between the forth- and back-flights was recorded, which indicated to me that the recorded East-West discrepancies could be due to radial accelerations of the earth's surface, which amounts to $1666 \mathrm{~km} / \mathrm{h}$ in the equatorial plane. Although I could not say that the remaining discrepancies were correlated with foreseeable impact of the respective directional accelerations, the recorded data clearly pointed to that possibility.

Note that - from the standpoint of an independent arbiter standing above the North pole, for instance, who does not rotate with the earth's surface - the Eastbound airplane would have 
flown at the speed of $2516=850+1666 \mathrm{~km} / \mathrm{h}$ relative to the arbiter, if the airplane would be flying over the equator, because the earth rotates eastwards. I assumed that the standard speed of most passenger airliners was $850 \mathrm{~km} / \mathrm{h}$. By the same token the Westbound airplane would have flown at the negative speed of $-816=850-1666 \mathrm{~km} / \mathrm{h}$ relative to the arbiter if the airplane would be flying over the equator. Effectively thus, both the Eastbound and the Westbound airplane would be seen by the arbiter as flying towards East, for the Westbound airplane was dragged by the earth's gravity toward East, as if on rear gear. In a sense, the Hafele-Keating experiments contradicted thus the naïve interpretation of Principle of Relativity (PR) that is traditionally misinterpreted as: If a train travels at a speed $v$ relative to embankment then one sitting inside that train could equally well say that the embankment appears as travelling at the speed $-v$ from the insider's point of view. I am not trying to bash Einstein but am reminding that guiding principles - as helpful as they may be when they are employed as just steppingstones - must not be viewed as reflecting scientific laws and thus be used for drawing scientific conclusions. Never substitute guiding principles for scientific laws.

Some authors who apply mathless formulations of physical laws outside of their intended context or the scope of their validity could inadvertently defy even experimentally confirmed facts such as the argument that by Newton's first law a motion at a constant velocity should cause no physical change in anything and thus dilation of time flow could not exist and therefore its presence is just a subjective perception that lies in the eyes of beholder [34].

Nevertheless, the need for an extension of the PR onto uniformly accelerated reference frames was briefly discussed in the STR setting in [35]. Principle of global relativity saying that inertial coordinates exist in spacetime has been proposed by Hestenes in [36]. He also proposed a local principle of relativity that I will not evaluate here not because I might disaagree with some ideas built upon the unwarranted SSR pparadigm, but because he invoked the traditional notion of 'covariance' which is based upon tensor calculus that I have shown to be flawed [29] and his local PR relies also on the traditinal 4D gradient operator, which I have reshaped in the multispatial framework built upon the MSR paradigm. Note that I have reformulated several traditional differential operators in a finegrained fashion [14] which act simultaneously on objects represented in both: the primary space and its dual reciprocal space [15] both of which form a certain abstract multispatial structure [16] and thus demand unrestricted division by zero [37] and new product differentiation rule [17]. These (only tangentially relevant) issues would require too much effort to tackle them here. Therefore, for the time being I shall propose univocal principle of relativity (UPR) based upon the aforesaid Hafele-Keating experiments. The UPR avows that any responsible use of the words 'relative' or 'relativity' in reference to motion should also specify the phrase "to what" and thus involve a comparison of the given motion with respect to yet another reference frame of an independent arbiter, which does not participate in the motion of the objects being compared, such as the arbiter standing above the North pole that does not participate in the motion of the earth's surface relative to which the speeds of those airplanes were compared.

The clearly infantile misinterpretation of the traditional PR leads to such illogical claims that in a Lorentz transformation the derivative $\partial t / \partial t^{\prime}$ is not the reciprocal of $\partial t^{\prime} / \partial t$ but equal to it and that there is allegedly no paradox [38]; compare also similar claim implicitly made in [39] which I have already debunked in [40]. Having said that let me affirm that making errors or mistakes is human but preventing others from proposing corrections and/or rectifications to be made to them, while at the same time tolerating tacitly veiled scientific misconceptions (and even covert lies) and promulgating nonsenses, suggests that a bias or even a thinly hidden adverse agenda is thriving in sciences, even in the mathematized ones. 
Before submitting my "experimental" paper [41] for publication I have called professors Hafele and Alley and Dr. Keating, asking them if there was anything that might disqualify their experiments. They kindly answered: No. Moreover, I have specifically asked prof. Hafele if he recorded the angles, directions, and time intervals during which the airplanes ascended after take-offs and then descended during landings. He told me that they did not record that kind of data because at that time there was no formula into which such data could be plugged in. Therefore, I have roughly emulated the possible impact of motion-related radial accelerations in [41], [42].

While I have practically reconciled the discrepancy recorded during the Westbound trip of the Hafele-Keating clocks that were only nominally flying Westwards, I could only partly reconcile the discrepancy recorded during the Eastbound trip. This very fact hinted at the possibility that perhaps some other than purely kinematic effects should also be taken into account, which led me to investigations of the possible impact of density of matter on gravity. That abstract (induced by mathematics) intuition guided by what I may call my "scientific instincts" also proved true [43] and has already been confirmed in several unbiased - yet formerly disregarded or just misinterpreted - experiments, see [21], [24], [25], [26].

While discussing Lorentz transformation in terms of some of its group properties (namely symmetry and transitivity) of an [unspecific] group Rindler virtually redefines dictionary when he asserts that "[...] the inverse of a Lorentz transformation is another Lorentz transformation ('symmetry') with parameter -v instead of v." - see [44] p.17. Using abstract groups without specifying their defining operation, which in that case of an additive group should pertain to obviously additive 'reversion' rather than to truly multiplicative 'inversion' that pertains to clearly multiplicative group operations, makes the unnecessary confusion possible. I have showed that multiplicative inversions are necessary for operating on dual reciprocal spatial structures [13], which in turn was needed to reveal the formerly concealed expanded product differentiation rule [17]. Without the latter rule the feasibility of pairing of dual reciprocal spaces [15] might become indefensible.

Furthermore, Rindler defines "[...] an ideal clock as one that is completely unaffected by acceleration, [...]" - see [44] p.27 and substantiates his conclusion that accelerations have no impact on the special-relativistic/kinematic dilation of time (which he admits as being real) by the conclusion drawn by Bailey who reported in [45] that they did not find evidence for accelerations affecting time dilation for muons travelling in circular orbits at high relativistic speeds inside the Muon Storage Ring at CERN. Yet since the relation between effective velocity $\mathrm{v}=\mathrm{R} \Omega$ and the angular velocity $\Omega$ obviously depends also on the radius $\mathrm{R}$ of the ring, I am not surprised that they could not detect the aforesaid impact at that resolution (measured in $\mu \mathrm{s})$ at CERN. But the significantly larger radius of the planet Earth, which I had to take into account while analyzing the formerly unreconciled and previously unexplained residual discrepancy (of $203 \mathrm{~ns}$ ) found and duly recorded during the Eastbound airplane flight of the Hafele-Keating experiment, apparently made also the conceptually possible impact of accelerations indismissible. Given the fact that Cesium clocks tick over 9 billion times per second one just cannot dismiss the discrepancy simply for no other reason than lack of mathematical formula that could have predicted the discrepancy.

Sagnac discovered that when the disk (with reflecting mirrors placed at the vertices of a quadrangle fixed to the disk) is rotated, the beam traveling in the rotation direction arrives at the source later than the beam traveling in the opposite direction (when the original beam is split by semitransparent mirror), which produces a shift of interference fringes. Recall that Sagnac-like frequency shift measured at radius $r$ on a rotating disk as compared to the frequency measured at the rotation axis has been confirmed experimentally again [46]. This 
kind of experimental evidence was either overlooked or just explained away in the past for some apologists apparently feared that it might contradicts a few principles upon which theories of relativity had been erected. Those experiments do not threaten to overthrow any of the theories of relativity but merely indicate that the STR should be enhanced by expanding it onto accelerations and consequently the radial-only GTR should be supplemented by extending it via the expanded STR as well as complemented via nonradial effects of gravity.

The reason for mentioning the experiments is that they implied the possibility of making enhancements to the STR and the need to include impacts of motion-dependent accelerations.

\section{DIFFERENTIAL GEOMETRY IN A NUTSHELL}

Newton's essentially geometric differential calculus ensued from his inherited realization that instantaneous speed - as the basically scalar magnitude of the effectively geometric notion of velocity - is tangent to the given trajectory curve at any point in the lengthbased space (LBS) of motion. Next, Leibniz algebraized the calculus making it thus much easier to deploy thoughtlessly and then a pleiade of mostly French mathematicians made remarkably abstract advances proudishly eliminating any picturesque references to the geometry that was so instrumental to Newton. Nevertheless, some scientists realized that there are also nuances indiscernible in the thoughtless algebraic approach that caused doubts and thus required conceptual thinking in geometric terms too. One of such nuances was the - purely conceptual at first - recognition that if an acceleration is needed to alter any initial velocity (including one that starts from zero at standstill), then any selfpropelled forward movement must resemble that of a twisting screw. This conclusion culminated in the splendid works of Frenet and Serret. I have already demonstrated that this still underappreciated - as it is in the inconsiderately pursued algebraic circles - deployment of the trihedron moving in the Frenet frame, resulted in some infantile and evidently embarrassing oversimplifications [27].

The screwlike movement of airplanes is clearly visible when they are making turns. I did read that only recently bioscientists "discovered" that sperm also moves like screws [89], even though it is over 170 years after the Frenet-Serret formulas have been published.

Hence a varying speed function - as the derivative of the radius vector $\mathbf{r}$ pointing to the trajectory curve with respect to the independently varying elapsing time parameter $t$ taken at a definite point in the length-based space of motion - must either cease to be the instantaneous magnitude of velocity that is tangent to the trajectory curve or the curve must be curving and twisting when certain higher differentials (i.e. acceleration and torsion) are applied. Hence, I have to stick to differential geometry and the choice to be made pertains to which methods shall I use. Leaving the coordinatefree tensor calculus aside due to its covert incompleteness [29], we are running out of options when it comes to examination of kinematic phenomena. Recall that anything that is unaltered by transformation of coordinates is invariant [47]. Exterior calculus models apply to systems for which the direction of change is given by a characteristic tangent vector while conventional calculus models apply to systems whose direction of change is arbitrary [48]. But like vector calculus, exterior calculus is too stiffy to offer truly realistic alternative to investigation of kinematic motion. What is left on the table is the trihedron moving along the given trajectory curve in the Frenet reference frame.

Frenet-Serret formulas are discussed in terms of angles in [49]. Curvature and torsion are discussed in terms of vectors in Frenet frame in [50], [51], [52], [53]. Curvature in terms of unit vectors on surfaces was discussed in [54]. Recall that torsion depends on $3^{\text {rd }}$ derivatives [of radius/position vector] - see [55], [53] or any other comprehensive textbook on differential geometry. Note that duality between curvature and torsion is analogous to the duality between electricity and magnetism [56]. Mappings of tangent spaces are discussed in [57]. 
Thus inside the 3D trihedron that moves along the given trajectory curve that is depicted within the Frenet frame $(\mathbf{T}, \mathbf{N}, \mathbf{B})$, the varying speed function $v_{s}=\frac{d s}{d t}$ whose magnitude equals to the value of the velocity vector, namely $v_{s}=\left|\boldsymbol{v}_{r}=\frac{d \boldsymbol{r}}{d t}\right|$, can, in general, be expressed as:

$$
\left\{\boldsymbol{v}_{r}=\boldsymbol{v}_{T}+\boldsymbol{v}_{N}+\boldsymbol{v}_{B}\right\} \Rightarrow v_{S}=\left|\boldsymbol{v}_{r}\right|=\sqrt{v_{T}^{2}+v_{N}^{2}+v_{B}^{2}}=\sqrt{v_{T}^{2}-\left(t_{N} a_{N}\right)^{2}-\left(t_{B} a_{B}\right)^{2}}
$$

where the $\mathbf{T}, \mathbf{N}, \mathbf{B}$, are mutually orthogonal unit vectors. The radius vector $\mathbf{r}$ is the pointing vector to the given trajectory curve $s$ and the speed components marked with $\mathrm{T}, \mathrm{N}, \mathrm{B}$, respectively, are visible inside of the $3 \mathrm{D}$ trihedron that comoves along the trajectory curve in the (tangential) T-direction, which trajectory can be curving in the (normal) N-direction, as well as twisting in the (binormal) B-direction within the sliding Frenet frame $(\mathbf{T}, \mathbf{N}, \mathbf{B})$ that is determined by the orthogonal unit vectors $\mathbf{T}, \mathbf{N}, \mathbf{B}$. There, the magnitude/value $a_{N}$ of normal acceleration $\boldsymbol{a}_{N}$ that acts during a certain elapsing "normal" time interval $t_{N}$ as well as the magnitude/value $a_{B}$ of a binormal acceleration $\boldsymbol{a}_{B}$ that acts during a certain elapsing "binormal" time interval $t_{B}$, are visible thus inside the moving trihedron of the Frenet frame.

Notice that if the motion that is determined by the arclength ds of trajectory curve $\mathrm{s}$ is not experiencing any selfpropelled (i.e. independent of an external force field) accelerations, that is: if the trajectory curve is neither curving nor twisting, then the motion - as seen inside the trihedron - maintains the conventional relationships expressed either in terms of velocities as $\boldsymbol{v}_{r}=\boldsymbol{v}_{T}$ or in terms of scalar speeds as $v_{S}=v_{T}$, of course. Nevertheless, even when the normal/curving acceleration is either nonexistent $a_{N}=0$ or temporarily inactive i.e. $t_{N}=0$ and thus the effective normal component $v_{N}=0$, the binormal/twisting acceleration still may be present twisting thus the straightlinear (in this case) trajectory. Hence motion depicted in Frenet frame can reveal more features than an external (to the motion) reference frame does. Caveat: Barrett O'Neill wrote: “The Frenet formulas are valid only for unit-speed curves; they tell the rate of change of the frame field T,N,B with respect to arc length. However, the speed $v$ of the curve is the proper correction factor in the general case." [58] p.70. Then he remarked that "Only for constant-speed curve is acceleration always orthogonal to velocity [...] In the general case, we analyze velocity and acceleration by expressing them in terms of the Frenet frame field." [58] p.71. The formula (2) has not only normal curvature but also binormal torsion that can twist the trajectory curve. For the trajectory is not only curved in the direction normal to but can also be twisted in the binormal direction with respect to the trajectory curve.

The reader may also compare the formula (2) to formwise similar expression though written in contravariant notation in [59] p.50. Given the fact that tensor calculus is valid only for purely radial phenomena for it tacitly precludes possible - and experimentally confirmed [21], [26] - presence of other than radial effects of purely radial force fields, I am refraining myself from relying on the flawed tensor calculus [29], but I respect the ingenuity and efforts of those who attained enviable conclusions working under the faulty SSR paradigm.

Some mathematicians and especially physicists apparently prefer to stick to the apodictic trend in mathematics that relies on the developments associated with such names as Gauss, Riemann, and Hilbert, to name just a few most relevant to our discussion. Although there is nothing wrong with what they did, the hype that surrounds their unquestionable achievements can sometimes be misleading. Gaussian curvature, for example, is directly associated with fundamental forms devised for surfaces, not curves [60], [61], [62], and therefore it could be aptly named "surfature" for lack of a better word. Since surface curvature vector is often defined as the sum of normal and tangential curvature vectors [63], I shall review those issues elsewhere. They could be used in numerical approximations but not really for 
drawing conclusions in conceptually unassailable theoretical investigations. Airplanes, for instance, fly along curves, not surfaces, and therefore their flights should better be analyzed first in terms of the Frenet frames whose trihedron moves along the trajectory curve, for otherwise some important aspects of their flights might be inadvertently overlooked. Although both Gauss and Riemann were contemporaries of Franet and Serret and thus might not have been influenced by the ideas of the latter two, today we do not really have such an excuse.

\section{SOME GEOMETRICAL PERCEPTIONS RELEVANT TO PHYSICS}

In [64] Elie Cartan does not specifically insist on absolute necessity to deploy the notions of curvature and torsion (in reference to the GTR). But he points out that the classical Riemannian space is without torsion and the space of absolute parallelism is without curvature [64]. He also admitted that one can introduce the notion of torsion into a Riemannian space with nonabsolute parallelism, but it would be difficult to do so in a general case [65]. Although the Cartan's remarks do not really disparage utilization of the Riemannian space, for elsewhere he also wrote that projective space is without torsion too [66] and the latter space is certainly helpful in some applications, his is polite admonition that perhaps more appropriate spatial structures for dealing with relativistic theories of gravitation should be sought somewhere else. I, for one, took his kind advice very seriously and spent a lot of time on developing my idea of paired dual reciprocal spatial structures, which he too inspired, before I decided to discard the simplistic traditional singlespatial geometries altogether. We should never ignore even smallest remarks made by such intellectual giants as Elie Cartan. For their imaginative conceptual intuition and structural insights might have saved countless scientists their precious time spent on pointless (in hindsight) pursuits, which they made while working under the formerly unrecognized and therefore unchallenged SSR paradigm.

I grew to disagree with some of E. Cartan's operational predilections, which were based on mappings, the commonly used tool for comparisons of operations under the former SSR paradigm, for it was pointed out that homomorphic groups are not always equivalent in the abstract sense, see [67]. But I certainly could not overemphasize E. Cartan's ingenious and farreaching structural instincts. For he also replaced the principle of duality of projective geometry by a principle of triality [68]; compare also [69]. Moreover, duality can also form antiisomorphism also called inverse isomorphism [70]. These achievements compelled me to investigate the alluring possibility that splitting the 4D spacetime into equidimensional 3D length-based space (LBS) and 3D time-based space (TBS) can be attained even from the old SSR-based premises, the feasibility of which I have already demonstrated in [40], and can be investigated and further advanced indeed. The latter feat prompted me to write this present paper ahead of other, more important to me, endeavors on my scientific to-do list.

The possible existence of 4D timespace as a counterpart of 4D spacetime has already been conjectured in [71], [72]. Yet in order to maintain the continuity and synchronization of the spacetime trajectory of fractalons, Ord invoked the use of a 3D timespace [73], which fact also gives some credibility to possible presence of a 3D TBS. Recall that timelike curves (all time at one point of space) and spacelike curves (all space at one instant of time) are dual notions [74]. While examining possible embedding functions, it was noticed that 6D line elements in both Kasner embedding signature (++- - - ) and Fronsdal embedding signature (+- - - -) there are compound $\mathrm{dz}$ elements, not just simple $\mathrm{dx}$ [75]. Thus we cannot discard the possibility of existence of other than purely radial components just because traditional mathematics did not recognize them. Lancini argued that since an electron is considered as a massless point-particle which moves in a spacetime with $(3+3)$ dimensions subjected to a field that attracts it towards the $(3+1)$ standard spacetime, the existence of spin and of magnetic 
moment indicates a rotation, hence motion in two dimensions. These two extra dimensions cannot be of a space kind (for Coulomb law would not behave as $\mathrm{r}^{-2}$ ) and therefore they must be two extra time dimensions [76]. Such conceptual reasonings are absolutely stunning. It was demonstrated that there is a physical role for a spacetime with two times when taken together with a gauged duality symmetry that produces appropriate constraints [77].

One can also argue that the largest number of dimensions $\mathrm{N}$ for which a vector space $\mathrm{V}_{\mathrm{NQ}}(\mathrm{Q}= \pm 1)$ over the reals (i.e. one that has Clifford algebra $\mathrm{C}_{\mathrm{NQ}}$ with the pseudoscalar $\mathrm{i}$ satisfying condition $\mathrm{i}^{2}=\mathrm{Q}$ ) gives some quite unique geometry is $\mathrm{N}=4$ (with corresponding $\mathrm{Q}=-$ 1) which uniquely defined geometry is one given by Minkowski metric [78]. That author offers thus mathematical condition [imposed] on the dimensionality and signature that makes it in some way unique among all possible candidates for a metric of spacetime and argues that the metric (-+++) and $(---+)$ give the same geometry, namely that of Minkowski spacetime [78]. Clifford remarked that the symbol $\mathrm{i}=\sqrt{ }(-1)$ is completely unintelligible so far as quantity is concerned. But if treated as a symbol of operation it has a perfectly clear and real meaning; thus any relation between complex numbers may be treated either as an algebraical fact relating to such numbers, or as a theorem concerning operations of turning and stretching unit steps [79]. In the latter sense the imaginary unit turned operator can be employed in the STR as well.

\section{OPERATIONAL EXPANSION OF CLASSIC STR ONTO ACCELERATIONS}

Since changing velocities do generate accelerations indeed, the latter should induce also certain emerging forces within the abstract mathematical force field that is usually depicted as the forceless 4D spacetime diagram, which nonetheless could be viewed as being quite independent of the surrounding us gravitational force field whose presence is due primarily to the physical existence of massive bodies. This issue shall be elaborated elsewhere.

In the special theory of relativity (STR) formulated in free space (i.e. in absence of force fields) the differential $\mathrm{d} \tau$ of the proper time interval $\tau$ that is measured by clocks placed inside trihedron of an inertial reference frame that moves at a certain constant speed $\mathrm{v}$ is given by

$$
d \tau=\frac{d t}{\gamma}=\sqrt{1-\beta^{2}} d t=\sqrt{1-\frac{v^{2}}{c^{2}}} d t=\frac{1}{c} \sqrt{c^{2}-v^{2}} d t
$$

where rapidity $\beta=\mathrm{v} / \mathrm{c}$, and gamma denotes the classic special-relativistic Lorentz factor

$$
\gamma=\frac{1}{\sqrt{1-\beta^{2}}}
$$

with dt denoting differential of the elapsing time parameter $t$ that is measured by clocks placed within the reference frame being at rest and $\mathrm{c}$ is the - assumed as being always constant in free space - speed of light in vacuum. Hence the relativistic 4velocity vector is expressed as

$$
w=\gamma\left(\frac{d x_{1}}{d t}, \frac{d x_{2}}{d t}, \frac{d x_{3}}{d t}, i c\right)
$$

see [80]. Einstein remarked that the special character of 4D continuum of physics is made by postulating that the fourth coordinate [that pertains to elapsing time] is purely imaginary whereas the others are real [81] in the mathematical sense of the term 'real'. For everything 
happens as if time were a fourth dimension of space [82] since time is actually defined by motion of 3D surface within 3D space [83]. For no matter what view we take on relativity, the physical methods of measuring time are quite different from those of measuring [distances in length-based] spaces [84]. In fact, intrinsically there is no dynamics; things begin to happen when we identify an [elapsing] time variable and slice spacetime into space and time-compare [85], which I have done in [40] because the 4D spacetime appears as an independent entity [86].

In the extensive though not really comprehensive (for it disregards other than purely radial effects of gravitational fields of the radial-only GTR) textbook on gravitation [59] on p.51 the authors disavowed the use of the imaginary term ict as the fourth dimension of spacetime but on the same page they have - inadvertently, I suppose - disclosed the actual reason for their dislike namely that "[...] the imaginary time coordinate hides from view the [declared as true] character of the geometric object being dealt with [...]" which is certainly true assessment for the ict was invented to avoid the unavoidable paradigm shift from the SSR to the MSR paradigm. But the authors also prevent the paradigm shift perhaps without realizing that at the time of that writing. For they use instead the fundamentally flawed tensor calculus [29] and endorse it as "The power of the geometric coordinate-free viewpoint [...]" see [59] p.64 that indeed conceals the actual multispatial mathematical and physical reality we live in, which is hidden beyond the incomplete reality they advocate as if it were true. They too defined an ideal clock that measures proper time, which should be so cleverly constructed as to depend not on any universal influence of acceleration on the march of time; for - according to them velocity produces a universal time dilation, but accelerations do not [59] p.393 and see also p.1055 therein. As I have mentioned above while on the subject of Bailey's experiments with muons conducted at CERN, no matter how precise they are, their conclusions derived from small scale (as compared with that of the earth) experiments and measured in $\mu$ s are incomparable with such large-scale experiments as Hafele-Keating and Alley conducted whose results were measured in ns, and thus cannot discredit the fact that accelerations had discernible and recorded impact on the accumulation of time by the atomic clocks flown around-the-world (as opposed to muons flying in the local ring around CERN).

Both the ict term and the coordinatefree tensor calculus conceal the formerly unrecognized multispatial character of the abstract mathematical- and consequently also that of the physical reality we live in. Nonetheless, while the ict obscures it inadvertently, due to suppression of previously prohibited division by zero, which is feasible [37] but without which performing realistic operations on infinities was impossible even though it is achievable [13], whereas tensor calculus tacitly conceals existence of the multispatial reality by presenting illegal operation, which covertly violates product differentiation rule, as admissible [29].

I have already demonstrated that the process of splitting the usual 4D spacetime into separate entities of space and time actually requires two equidimensional 3D spaces [40]. If so, then the STR should also be operationally expandable (and thus structurally extendable as well) so that not only the usual spatial terms can be cast within the conventional 3D reference frame, but it should also be possible to depict the temporal terms inside a 3D reference frame portrayed in terms of length-based space.

Since both differentials $\mathrm{d} \tau$ and $\mathrm{dt}$ of the two (respective moving and resting) elapsing time parameters can be counted, the differential $\mathrm{d} \tau$ of the proper/moving time $\tau$ and the differential $\mathrm{dt}$ of the usual/resting time $\mathrm{t}$, respectively, are regarded in the purely operational formula (3) as scalar magnitudes (as opposed to a possible structural/geometric formula to be offered elsewhere). It is easy to see that the equation (3) in conjunction with the formula (2) that applies to the trihedron comoving along the given trajectory curve, could be operationally expanded 
onto scalar magnitudes of mutually orthogonal accelerations (that are visible inside the comoving trihedron) for essentially nonrotational motion and thus could be rewritten as

$$
d \tau=\frac{1}{c} \sqrt{c^{2}-v_{T}^{2}-v_{N}^{2}-v_{B}^{2}} d t=\frac{1}{c} \sqrt{c^{2}-v_{T}^{2}-\left(t_{N} a_{N}\right)^{2}-\left(t_{B} a_{B}\right)^{2}} d t
$$

if the elapsing time flows in the radial dimension, of course. Obviously, the scalar velocity magnitude (also known as speed) $v_{T}=d s / d t_{T}$ is taken in the tangential direction and thus the differential ds of the arclength of the given trajectory path $\mathrm{s}$ is differentiated with respect to the tangentially accumulated elapsing time component $\mathrm{dt}_{\mathrm{T}}$ of the usual, total elapsing time parameter t, of course. In other words: the scalar velocity (i.e. speed) $v$ that appears in the traditional special-relativistic formula (3) is actually the tangential component of the speed standing in the expanded special-relativistic formula (6), namely: $v_{T}=d s / d t_{T}$. The latter identification adds nothing really new, for constant velocity is always represented as vector tangent to the trajectory curve pointed to by the radius vector $\mathbf{r}$ compare [87]. What I have added is the - straightforward as they are in differential geometry - evaluations of the normal and binormal subcomponents of the total speed $v$ in terms of the respective directional accelerations, namely: $v_{N}=t_{N} a_{N}$ and $v_{B}=t_{B} a_{B}$ which are mutually orthogonal so that (due to their orthogonality within the trihedron) the total speed standing in (3) is actually composed as shown in the formula (2), provided it is considered and evaluated within that trihedron that comoves, turns and/or twists along the trajectory curve just as the curve does.

Recapitulation: under the assumption that total elapsing time flows in the radial dimension along the radius vector $\mathbf{r}$ (also known as position vector) that points to the given trajectory curve s, when the velocity vector equals to the derivative $\boldsymbol{v}_{\boldsymbol{r}}=\frac{d \boldsymbol{r}}{d t_{r}}$ with respect to the radially accumulated time parameter $t_{r}$, the increase (or decrease) in magnitude of the total velocity vector, whose tangential component's magnitude is $\left|v_{T}\right|=v_{T}=\frac{d s}{d t_{T}}$, should be influenced also by the magnitude of the normal acceleration $a_{N}=\left|\boldsymbol{a}_{N}\right|$ acting over the elapsing time interval $t_{N}$ that is accumulated along the normal direction with respect to the given trajectory curve s. Similarly, for the radially accumulated elapsing time parameter $t_{r}$, the increase (or decrease) in magnitude of the total velocity vector, whose tangential component's magnitude is $\left|\boldsymbol{v}_{T}\right|$, should be influenced also by the magnitude of binormal acceleration $a_{B}=$ $\left|\boldsymbol{a}_{B}\right|$ acting over the elapsing time interval $t_{B}$ that is accumulated along the binormal direction with respect to the trajectory curve s. Note that only in a 2D plane normal acceleration may suffice. But in 3D setting both normal and binormal accelerations should be considered in general. Compare also [80], p.14f for even the traditional special-relativistic representation of proper time (which does not contain any accelerations) corresponds to the formula (6) conceptually.

While it is not absolutely necessary, this latter reference is made in order to satisfy the (common sense) requirement that a new theory must be complementary to the ordinary 4D relativity unifying new concepts and capable of explaining experimentally well-established facts [88]. For the formula (6) in conjunction with the equidimensional split of 4D spacetime into 3D space and 3D time [40] virtually indicates the possibility that eventually the STR could be formulated in a 6D fashion, regardless of whether one likes it or not - compare also [71], [72]. For mathematics should not be subject of some arbitrary preconceived ideas but its structural (that is geometric and quasigeometric) features must be imposed by unrestricted operational procedures, which may be altered after subsequent shifts of paradigms. This assertion is the essence of my new synthetic approach to mathematical and mathematized 
theories, such as theories of mathematical physics. Neither mathematical nor physical theories are written in stone and therefore their defiant defense against innovations cannot stand for ever. Mathematics should be continuous work in progress preferably guided by results of unbiased experiments.

Since in order to account for a varying speed function one needs to apply accelerations or decelerations, the formula (6) should not come as a surprise. Otherwise, one would really need some kind of mathemagic to make the traditional Einstein's STR at least operationally (if not structurally yet) complete. I am not blaming Einstein, who certainly did his best, but his apologists who teach that the GTR took care of accelerations are either brain dead or insulting the intelligence of their students. For the basically kinematic effects of the STR that can surely act in other than radial (hence tangential/normal/binormal) directions are independent of the purely radial effects of the gravitational force field. For selfpropelled airplanes, for instance, do not fly directly toward the center of the Earth (nor away from it) but practically along its surface, unless they are going to crash.

\section{CONCLUSIONS}

Although I have already proposed a simpler formula than (6) endowed with single combined acceleration [42], which I have used to partly explain the previously unexplained residual data acquired during the Eastward trip of atomic clocks flown around-the-world (and to fully explain the Westbound trip) [41], that formula earned me virtual censorship for well over 12 years and delayed the rectification of the STR, whose suspension allowed the still ongoing fooling of students everywhere for well over 30 years with total impunity.

However, the formula (6) is too simple and too obvious - at least from the standpoint of differential geometry - to be discounted. It has been defined for any length-based space that includes the quasispatial structure of 4D spacetime and is implemented within the Frenet trihedron that comoves along the given trajectory curve in absence of direct interactions with external force fields.

The Hafele-Keating experiments with atomic clocks flown around-the-world aboard commercial airplane virtually confirmed the necessity of employing accelerations in order to account for the East-West elapsing time asymmetry found by both Hafele as well as by Alley who conducted similar experiments. Since selfpropelled airplanes fly under the influence of gravity and of thrust of their engines, the formula (6) is really necessary in order to account for the impact of their engines on the trajectory curve.

Although it was presumed that velocity vector is always tangential to the trajectory curve, which is true when looking at the velocity vector from an external reference frame, this not exactly true from an internal reference frame of the trihedron - sometimes identified with the Frenet frame - that not only comoves with the point traveling along the trajectory (which point may be identified with the origin of the regarded as rigid trihedron), but also turns and twists just as the trajectory curve does. While accepting the proven Frenet-Serret kinematics, former mathematics and physics never went beyond the fact that when zero-dimensional point moves along the given trajectory curve, one cannot see that the point should turn and/or twist in the normal and binormal directions with respect to the trajectory curve. This was hideously clever deception frivolously justified by the fact that point has zero dimensions. Yet when the point is regarded as the origin of the rigid trihedron that moves and could turn (in its normal direction) and could twist (in its binormal direction) then one must admit that both normal and binormal velocities could reveal their presence within the internal reference frame of the trihedron comoving with the trajectory curve. By the same token, the above reasoning that pertains to internal subcomponents of velocity can also be applied to respective normal and 
binormal accelerations. The term 'comoving' conveys more than the phrase "moving along", for it implies also possible turning and twisting that the trajectory curve exhibits, which may be observed in mathematical software packages.

Operationally, the formula (6) expands special relativity onto purely kinematic (i.e. independent of the surrounding gravitational field) accelerations. Structurally, it could extend special relativity into geometric and quasigeometric spatial structures. Thud the formula (6) is needed to account for kinematical aspects of selfpropelled motion and for conceptual completeness of the classic special relativity. The formula (6) does not contradict any mathematically reasonable theory of contemporary physics. In particular, it is not flouting any mathematically sound theories; neither the Einstein's Special Theory of Relativity (STR) nor his General Theory of Relativity (GTR) is defied by the - operationally expanded onto accelerations - formula (6). As a matter of fact, the formula (6) obviously complements the clearly incomplete classic STR and thus supplements also the GTR, which is only a radial theory of gravitation, not really the truly general theory of gravitational phenomena that should include also nonradial gravitational effects, which too are generated even by purely $\mathrm{radial} / \mathrm{center}$-bound force fields.

\section{ACKNOWLEDGEMENTS}

I am greatly indebted to late Professor Hans-Jürgen Treder who, as the director of the Einstein lab in Germany in the 1980s, had both the scientific ability and the intellectual courage to see the point I made based upon my partial reconciliation of the Hafele-Keating experiments with atomic clocks flown around-the-world aboard commercial airplane.

Many thanks to the journal's editors and referees for their constructive criticism, comments, advices, and recommended revisions, all of which are highly appreciated. Besides revising the text on the recommendations, I have added also the following Appendix:

\section{APPENDIX: THE PROPOSED EXPANSION IS JUSTFIED BY EXPERIMENTS}

In mathematics there are three kinds of existence: empirical, definitional, deductive [90]. I have addressed all three of them, by showing the empirical justification of the eq. (6) that came from the Hafele-Keating experiments, have defined them mathematically and have deduced them from special relativity. The justification is incomplete on purpose in this paper, because the experimenters did not collect data about speeds or angles of the flights. In the next paper on the topic, I have included the third component of the total acceleration (which did not emerge from the experiments. Hence the total complete formula offered in the next paper is a conjecture whereas the incomplete formula (6) is rooted in the experiments. In other words: due to lack of pertinent experiments the - experimentally implied formula (6) - is incomplete, and if it were complete (as the one proposed in the next paper) it would be only mathematical conjecture not justified by directly relevant experiments.

This paper offers new formula inspired and justified by the briefly reviewed experiments. The incomplete (two-component acceleration-dependent) formula is experimentally justified by reconciling the discrepancy between predicted and recorded elapsing time accumulated by the atomic clocks during the westbound flight of the airplane carrying the atomic clocks in the Hafele-Keating experiment.

There are two kinds of analyses [in applied mathematics]: 1) the problem to prove, and 2) the problem to find something unknown [91]. This paper offers an accelerations-dependent relativistic formula, which was unknown before I had devised it upon experimental hints. No 
proof was intended to be offered in this paper because proofs can only acknowledge consistency of derivations, not really truth of the proposed formulas, whose justification must thus be supplied by the Nature in unbiased experiments. For mathematical objects exist as normative systems [92]. Existence means thus that a mathematical object belongs to a mathematical system of objects and relations [93]. Thus, for some thinkers the problem of existence of the primary entities of mathematics is analogous to the problem of existence of the king of chess or the ace of spades [94] and so the "reality" of the existential problems [in mathematics ...] is either profoundly real or absurdly surreal [95].

For [pure/theoretical] mathematicians, a mathematical entity exists provided there is no contradiction implied in its definition, either in itself or with the previously admitted [96]. Hence the two fundamental conditions of existence of abstract objects, which insist that object should be well defined and free from internal contradictions [97] are insufficient; only experiments could justify - but not prove existence of mathematical entities. Some authors claim that mathematical concepts are well-defined (uniquely and unambiguously) if two mathematicians talk about the same thing [98], whereas others believed that today's integral geometry is characterized by generalization of its concepts combined with their reality [99]. Thus, mathematical propositions and theories are exact in a sense in which empirical propositions and theories are not; and mathematical theories are existential in a sense in which - in many senses of 'the term] 'logic' - logic is not [100]. Bertrand Russell believed that mathematics is concerned not with (physical) existence but only with the possibility of existence [101]. Brouwer wanted to give up all existence proofs whereas Hilbert wanted a systematic way to show that they were unnecessary in principle [102]. Hence the need for experimental justification emerged in applied mathematical theories dealing with phenomena grounded in the physical reality. For Jammer remarked that formidable scientific achievements of the past may not be quite (or entirely) true within the present framework of sciences. Space as conceived by Newton proved to be an illusion, but for [most] practical purposes a very fruitful illusion [103]. Helmholtz admonished us that when meanings of concepts change with time, they must be updated [104]. I have showed that the path-independence of work done theorem is invalid in center-bound force fields [105], for example, which has already been politely acknowledged by Greenleaf [106].

By disregarding the new mathematics that is guided by experimental hints, but sticking to the traditional mathematics, a misguided physical experiment has allegedly found that the diameter of hydrogen proton allegedly shrinks by about $4 \%$ when its electron is replaced by muon [107], which was nicely explained in [108]. If you check the mathematics used in the supplemental material attached to [107], you can see that they use the radial scalar potential function whose mathematical core is $\mathrm{V}=1 / \mathrm{r}$ not the fully evaluated $3 \mathrm{D}$ potential that pays for the work done by the given force field. I have shown in [109] that an error of at least $3.48 \%$ is to be expected due to mathematical evaluation alone, not to mention some physical constraints that could further increase the discrepancy. Some authors explicitly disparaged and thus virtually discouraged the use of moving trihedron in Frenet reference frames [110], without which it may be difficult to recognize the possible presence of other than purely radial (i.e. nonradial) effects of the radial/center-bound force fields.

Proofs are derivations from axioms and primitives. Einstein invented special relativity on the basis of principle of relativity and assumed constancy of speed of light in vacuum [111]. He did not prove his theory but justified its predictions by experiments, some of which had performed Lorentz and others [112]. So is the expanded relativistic formula (6) also justified by experiments. Theories of physics are not intrinsically axiomatic. They could be disproved or justified by experiments but cannot be proved a priori arising from selfevident axioms. 


\section{REFERENCES}

[1] Kauffman S. \& Smolin L. Combinatorial dynamics and time in quantum gravity. [pp.101129 in: Kowalski-Glikman J. (Ed.) Towards quantum gravity. Berlin: Springer, 2000, p.117].

[2] DeWitt B.S. Spacetime as a sheaf of geodesics in superspace. [pp.359-374 in: Carmeli M., Fickler S.I. and Witten L. (Eds.) Relativity. New York: Plenum Press, 1970].

[3] Kar K.C. Notes on the theory of relativity. Ind. J. Theor. Phys. 17(1) (1969) 1-11

[4] Kar K.C. \& Dutta C. On the theory of relativity. Ind. J. Theor. Phys. 16(1) (1968) 1-6

[5] Duhem P. The aim and structure of physical theory. Princeton, NJ: Princeton U. Press, 1991, p.193.

[6] Zwillinger D. Handbook of differential equations. Boston: Academic Press, 1989, pp.68,69.

[7] Ulam S.M. Problems in modern mathematics. First published under the title A collection of mathematical problems. New York: Wiley, 1960, p.63ff.

[8] Glasko V.B. Inverse problems of mathematical physics. New York: AIP, 1988, p.8.

[9] Lavrent'ev M.M., Romanov V.G. \& Shishatskiǔ S.P. Ill-posed problems of mathematical physics and analysis. Providence, RI: AMS, 1986, pp.V, 2.

[10] Lavrentiev M.M. Some improperly posed problems of mathematical physics. Berlin: Springer, 1967, p.2.

[11] Airapetyan R.G. \& Ramm A.G. Dynamical systems and discrete methods for solving nonlinear ill-posed problems. [pp.491-536 in: Annastassiou G.A. (Ed.) Applied mathematics review I. Singapore: World Scientific, 2000, see p.492 and refs. therein].

[12] Beck J.V., Blackwell B. \& St. Clair C.R., Jr. Inverse heat conduction. Ill-posed problems. New York: Wiley, 1985, pp.3ff,7.

[13] Czajko J. Multiplicative inversions involving real zero and neverending ascending infinity in the multispatial framework of paired dual reciprocal spaces. World Scientific News 151 (2021) 1-15 http://www.worldscientificnews.com/wp-content/uploads/2020/10/WSN151-2021-1-15.pdf

[14] Czajko J. Finegrained 3D differential operators hint at the inevitability of their dual reciprocal portrayals. World Scientific News 132 (2019) 98-120

http://www.worldscientificnews.com/wp-content/uploads/2019/06/WSN-132-2019-98120.pdf

[15] Czajko J. Dual reciprocal scalar potentials paired via differential operators in Frenet frames make the operators to act simultaneously in each of two paired 3D reciprocal spaces. World Scientific News 137 (2019) 96-118

http://www.worldscientificnews.com/wp-content/uploads/2019/09/WSN-137-2019-96118.pdf

[16] Czajko J. Pairing of infinitesimal descending complex singularity with infinitely ascending, real domain singularity, World Scientific News 144 (2020) 56-69

[17] Czajko J. New product differentiation rule for paired scalar reciprocal functions. World Scientific News 144 (2020) 358-371

researchgate.net/publication/340536056_New_product_differentiation_rule_for_paired _scalar_reciprocal_functions

[18] Coleman M.P. An introduction to partial differential equations with Matlab ®. Boca Raton, FL: Chapman \& Hall/CRC, 2005, pp.114,403.

[19] Pinsky M.A. Partial differential equations and boundary-value problems with applications. New York: McGraw-Hill, 1991, pp.166f,200,209ff,234. 
[20] Schey H.M. Div, grad, curl, and all that. An informal text on vector calculus. New York: Norton, 1997, p.125ff.

[21] Czajko J. On Conjugate Complex Time II: Equipotential Effect of Gravity Retrodicts Differential and Predicts Apparent Anomalous Rotation of the Sun. Chaos Solit. Fractals 11 (2000) 2001-2016

[22] Czajko J. Path-independence of work done theorem is invalid in center-bound force fields. Studies in Mathematical Sciences 7(2) (2013) 25-39 http://cscanada.net/index.php/sms/article/view/j.sms.1923845220130702.2469/5279

[23] Czajko J. Equipotential energy exchange depends on density of matter. Stud. Math. Sci. 7(2) (2013) 40-54 http://cscanada.net/index.php/sms/article/view/j.sms.1923845220130702.2752/5336

[24] Czajko J. On the Intrinsic Gravitational Repulsion. Chaos Solit. Fractals 19 (2004) 683700

[25] Czajko J. Equalized mass can explain the dark energy or missing mass problem as higher density of matter in stars amplifies their attraction. World Scientific News 80 (2017) 207$238 \mathrm{http} / / / \mathrm{www} . w o r l d s c i e n t i f i c n e w s . c o m / w p-c o n t e n t / u p l o a d s / 2017 / 07 / \mathrm{WSN}-80-2017$ 207-238.pdf

[26] Czajko J. Galilei was wrong: Angular nonradial effects of radial gravity depend on density of matter. Int. Lett. Chem. Phys. Astron. 30 (2014) 89-105 https://www.scipress.com/ILCPA.30.89

[27] Czajko J. Radial and nonradial effects of radial fields in Frenet frame. Applied Physics $\begin{array}{llll}\text { Research } & 3(1) & \text { (2011) } & \text { 2-7 }\end{array}$ http://www.ccsenet.org/journal/index.php/apr/article/viewFile/9555/7421 or https://pdfs.semanticscholar.org/1568/4da520085028442dbd46308bfd46c0e8c195.pdf

[28] Czajko J. With Equalized Mass, its Density of Matter can Affect Radial Gravitational Interactions Too. Int. Lett. Chem. Phys. Astron. 54 (2015) 112-121

https://www.scipress.com/ILCPA.54.112

[29] Czajko J. Flawed fundamentals of tensor calculus. World Scientific News 149 (2020) 140165 researchgate.net/publication/344399810_Flawed_fundamentals_of_tensor_calculus

[30] Lawrie I.D. A unified grand tour of theoretical physics. Bristol: Adam Hilger, 1990, p.43.

[31] Hafele J.C. \& Keating R.E. Around-the-World Atomic Clocks: Predicted Relativistic Time Gains. Science 177 (4044) (1972) 166-168

[32] Hafele J.C. \& Keating R.E. Around-the-World Atomic Clocks: Observed Relativistic Time Gains. Science 177 (4044) (1972) 168-170

[33] Alley C. P. Proper Time Experiments in Gravitational Fields with Atomic Clocks, Aircraft, and Laser Light Pulses. [pp. 363-427 in: Meystre P. \& Scully M. O. (Eds.) Quantum Optics, Experimental Gravity, and Measurement Theory. New York: Plenum, 1983].

[34] Katti A.N. The mathematical theory of special and general relativity. North Charleston, SC: CreateSpace Independent Publishing, 2013, p.20f.

[35] Hawking S. (Ed.) On the shoulders of giants. Selections from The Principle of Relativity by Albert Einstein. Philadelphia, PA: Running Press, 2002, p.35ff.

[36] Hestenes D. Space-time algebra. New York: Gordon and Breach, 1966, p.74.

[37] Czajko J. Unrestricted division by zero as multiplication by the - reciprocal to zero infinity. World Scientific News 145 (2020) 180-197

http://www.worldscientificnews.com/wp-content/uploads/2020/04/WSN-145-2020180-197.pdf

[38] Fock V. The theory of space, time and gravitation. New York: Macmillan, 1964, p.42ff. 
[39] Freitag E. Riemannian and Lorentzian geometry. Differential geometry, Riemannian manifolds, Lorentzian manifolds, cosmology. Coppell, TX: 2020, p.80ff.

[40] Czajko J. Splitting 4D spacetime into 3D space and 1D time involves operationally needed presence of two 3D spaces enclosed inside two 4D paired dual reciprocal spatial structures. World Scientific News 152 (2021) 82-110

http://www.worldscientificnews.com/wp-content/uploads/2020/12/WSN-152-2021-82110.pdf

[41] Czajko J. On the Hafele-Keating experiment. Ann. Phys. (Leipzig) 47 (1990) 517-518

[42] Czajko J. Experiments with flying atomic clocks. Exper. Tech. Phys. 39 (1991) 145-147 https://www.researchgate.net/publication/320812632_Experiments_with_Flying_Atomi c_Clocks

[43] Czajko J. On Conjugate Complex Time I: Complex Time Implies Existence of Tangential Potential that Can Cause Some Equipotential Effects of Gravity. Chaos Solit. Fractals 11 (2000) 1983-1992

[44] Rindler W. Introduction to special relativity. Oxford: Clarendon Press, 1991, p.13ff.

[45] Bailey J. et al. Measurements of relativistic time dilation for positive and negative muons in a circular orbit. Nature 268 (1977) 301-305

[46] Hay H.J. et al. Measurement of the red shift in an accelerated system using the Mössbauer effect in $\mathrm{Fe}^{57}$. Phys. Rev. Lett. 4(4) (1960) 165-166

[47] Veblen O. \& Whitehead J.J.C. The foundations of differential geometry. Cambridge: Cambridge Univ. Press, 1960, p.46.

[48] Story T.L. Introduction to differential geometry with applications to Navier-Stokes dynamics. New York: iUniverse, 2005, p.74.

[49] Graustein W.C. Differential geometry. Mineola, NY: Dover, 2006, p.38ff.

[50] Gray A., Abbena E. \& Salamon S. Modern differential geometry of curves and surfaces with Mathematica ®. Boca Raton, FL: Chapman \& Hall/CRC, 2006, p.195ff.

[51] Banchoff T. \& Lovett S. Differential geometry of curves and surfaces. Natick, MA: A.K. Peters, 2010, p.68ff.

[52] Sochi T. Introduction to differential geometry of space curves and surfaces. Middletown, DE, 2017, pp.47ff, 110ff.

[53] Stoker J.J. Differential geometry. Delhi: Chaudhury, 2014, pp.54f,100ff.

[54] Guggenheimer G. W. Differential geometry. New York: Dover, 1977, pp.157ff,206ff.

[55] Young E.C. Vector and tensor analysis. New York: Marcel Dekker, 1978, p.106f.

[56] Kühne R.W. Cartan's torsion: Necessity and observational evidence. [pp.37-42 in: Dvoeglazov V. \& Garrido A.E. (Eds.) Relativity, gravitation, cosmology. New York: Nova Science, 2004].

[57] Doolin B.F. \& Martin C.F. Introduction to differential geometry for engineers. Mineola, NY: Dover, 2012, p.23ff.

[58] O’Neill B. Elementary differential geometry. San Diego: Academic Press, 1997, p.70ff.

[59] Misner C.W., Thorne K.S. \& Wheeler J.A. Gravitation. New York: Freeman, 1973, pp.50,51,64,393,1055.

[60] Wilson P.M.H. Curved spaces. From classical geometries to elementary differential geometry. Cambridge: Cambridge Univ. Press, 2008, p.153ff.

[61] McCleary J. Geometry from a differentiable viewpoint. Cambridge: Cambridge Univ. Press, 1994. p.139ff.

[62] Stillwell J. Geometry of surfaces. New York: Springer, 1992, p.75ff.

[63] Struik D.J. Lectures on classical differential geometry. New York: Dover, 1988, p.127ff.

[64] Cartan É. Selecta. Paris : Gauthier-Villars, 1939, p.24. 
[65] Cartan E. Notice sur les travaux scientifiques. Paris : Gauthier-Villars, 1974, p.118.

[66] Cartan E. Sur les formes differentielles en géométrie. [pp.905-907 in : Cartan E. CEuvres complètes t.1 p.2. Paris : Gauthier-Villars, 1955, see p.907].

[67] Zassenhaus H.J. Theory of groups. New York: Chelsea, 1958, p.35ff.

[68] Cartan E. Le principe de dualité et la théorie des groupes simples et semi-simples. [pp.555568 in: Cartan E. Euvres complètes I. Paris : CNRS, 1984, see p.567].

[69] Cartan E. Le principe de dualité et certaines intégrales multiples de l'espace tangentiel et de l'espace réglé. [pp.265-302 in Cartan E. Oeuvres complètes. T.1 p.2. Paris : GauthierVillars, 1953].

[70] Lautman A. Symétrie et dissymétrie en mathématiques et la physique. [pp.54-65 in : Le Lionnais F. Les grands courants de la pensée mathématique. Paris : Editions des « Cahiers du Sud », 1948].

[71] Czajko J. Operational constraints on dimension of space imply both spacetime and timespace. Int. Lett. Chem. Phys. Astron. 36 (2014) 220-235 https://www.scipress.com/ILCPA.36.220

[72] Czajko J. Operational restrictions on morphing of quasi-geometric 4D physical spaces. Int. Lett. Chem. Phys. Astron. 41 (2015) 45-72 https://www.scipress.com/ILCPA.41.45

[73] Ord G.N. Fractal space-time: a geometric analogue of relativistic quantum mechanics. $J$. Phys. Math. Gen. 16 (1983) 1869-1884

[74] Geroch R. \& Horowitz G.T. Global structure of spacetimes. [p.212-293 in: Hawking S.W. \& Israel W. (Eds.) General relativity. An Einstein centenary survey. Cambridge: Cambridge Univ. Press, 1979, see p.243].

[75] Fujitani T., Ikeda M. \& Matsumoto M. On the imbedding of the Schwarzschild space-time I. J. Math. Kyoto Univ. 1-1 (1961) 43-61

[76] Lanciani P. A model of the electron in a 6-dimensional spacetime. Found. Phys. 29(2) (1999) 251-265

[77] Bars I., Deliduman C. \& Andreev O. Gauged duality, conformal symmetry, and spacetime with two times. Phys. Rev. D 58 (1998) 066004

[78] Lemos P.J. On the mathematical uniqueness of Minkowski space. Eur. J. Phys. 4 (1983) $1-2$

[79] Clifford W.K. The common sense of the exact sciences. New York: Dover, 1955, pp.172, 175.

[80] Sommerfeld A. Lectures on theoretical physics. I: mechanics. New York: Academic Press, LCCCN 50-8749 (undated), p.14ff.

[81] Einstein A. Die Kompatibilität der Feldgleichungen in der einheitlichen Feldtheorie. Sitz.ber. Preuss. Ak. Wiss. 1929 pp.18-23

[82] Poincaré H. Mathematics and science. Last essays. New York: Dover, 1963, p.23.

[83] Sheeter S. The unified model of the universe. Berkeley, CA: Process Press, 1980, p.47.

[84] Conway A.W. The quaternionic form of relativity. Phil. Mag. 24 (1912) 208

[85] Ashtekar A. Canonical quantum gravity. [pp.189-220 in: Mann R. \& Wesson P. (Eds.) Gravitation. A Banff Summer Institute. Singapore: World Scientific, 1991].

[86] Einstein A. \& Infeld L. On the motion of particles in general relativity theory. Can. J. Math. 209 (1949) 1

[87] Stewart J. Calculus. Early vectors. Belmont, CA: Brooks/Cole Publishing, 1999, p.140.

[88] Hatzikonstantinou P. \& Moyssides P.G. Comments on a six-dimensional space-time scheme. Phys. Lett. A 140 (1989) 85-89, see p.88f.

[89] Gadelha H. The beautiful spirals of sperm cells on the move. (as of July 21, 1020) https://www.sciencefriday.com/segments/sperm-3d-motion/ 
[90] Katz R. Axiomatic analysis: An introduction to logic and the real number system. Boston: D.C. Heath \& Co., 1964, p.32ff.

[91] Hintikka J. \& Remes U. The method of analysis. Dordrecht: D. Reidel, 1974, p.119.

[92] Panov M.I. Methodological problems of the development and application of mathematics. Moscow, 1985, p.25, [in Russian].

[93] Freytag-Löringhoff B. von. Philosophical problems of mathematics. New York: Philosophical Library, 1951, p.30.

[94] Goodstein R.L. Existence in mathematics. Compos. Math. 20 (1968) 70.

[95] Fang J. Mathematical existence. Philos. Math. 11 (1974) 5-8.

[96] Poincaré H. Science and hypothesis. New York: Dover, 1952, p.44.

[97] Janiszewski Z. Sur le réalisme et l'idéalisme en mathématiques, in: Janiszewski Z. Oeuvres choisies. Warszawa: PWN, 1962, pp. 309-317.

[98] Borel E. L'imaginaire et le réel en mathématiques et en physique. Paris : Albin Michel, 1952, p.99.

[99] Comte A. La synthèse subjective ou système universel des conceptions propres á l'état normal de l'humanité I. Paris, 1900, p.508.

[100] Körner S. The philosophy of mathematics. An introductory essay. London: Hutchinson Univ. Library, 1968, p.58.

[101] Benacerraf P. \& Putnam H. (Eds.) Philosophy of mathematics. Englewood Cliffs: Prentice-Hall, 1964, p.6.

[102] Beeson M. Constructivity in the nineties. [pp.127-144 in: Czermak J. (Ed.) Philosophy of mathematics I. Vienna: Verlag Hölder-Pichler-Tempsky, 1993].

[103] Jammer M. Concepts of space. The history of theories of space in physics. New York: Dover, 1993, p.173.

[104] Helmholtz H. Vorlesungen über theoretische Physik I. Leipzig: Barth, 1903, p. 2.

[105] Czajko J. Path-independence of work done theorem is invalid in center-bound force fields. Stud. Math. Sci. 7(2) (2013) 25-39

http://cscanada.net/index.php/sms/article/view/j.sms.1923845220130702.2469/5279

[106] Greenleaf F.P. Introduction to complex variables. Philadelphia: Saunders, 1972, p.105.

[107] Antognini A. et al. Proton structure from the measurements of 2S-2P transition of muonic hydrogen. Science 339/6118 (2013) 417-420

[108] Margolis H.S. How big is the proton? Science 339/6118 (2013) 405-406

[109] Czajko J. Linear magnitudes estimated via energy were likely overestimated by over 3.48\%. Int. Lett. Chem. Phys. Astron. 32 (2014) 32-41 https://www.scipress.com/ILCPA.32.32

[110] Sternberg S. Curvature in mathematics and physics. Mineola, NY: Dover, 2012, p.79.

[111] Einstein A. On the electrodynamics of moving bodies. [pp.35-65 in: Lorentz H.A. et al. (Eds.) The Principle of Relativity. New York: Dover, 1923, see p.41].

[112] Lorentz H.A. Electromagnetic phenomena ... [pp.9-34 in: Lorentz H.A. et al. (Eds.) The Principle of Relativity. New York: Dover, 1923, see p.33f]. 